\title{
Mechanisms of Costimulation
}

\author{
Arlene H. Sharpe ${ }^{1}$ \\ ${ }^{1}$ Departments of Pathology, Harvard Medical School and Brigham and Women's Hospital, \\ Boston MA USA
}

The immune system has the remarkable ability to defend against a diversity of microbial pathogens, yet not respond to self. The discovery of the T-cell antigen receptor (TCR) provided an explanation for the specificity and diversity of T-cell responses. Yet, functional studies of Lafferty and colleagues (1) suggested that antigen alone was not sufficient to drive the activation of naive $\mathrm{T}$ cells and led to the two signal concept of T-cell activation. According to this model, productive T-cell activation requires a first signal provided by the interaction of antigenic peptide/major histocompatibility complex (MHC) with the TCR and a second, antigen-independent co-signal, the 'costimulatory signal'. In addition, seminal studies of Jenkins and Schwartz $(2,3)$ demonstrated that TCR-mediated activation of T cells in the absence of costimulation resulted in antigen-specific unresponsiveness (termed T-cell anergy), rendering the $\mathrm{T}$ cells unable to respond to subsequent exposure to antigen. Thus, costimulation was postulated to have a pivotal role in determining whether the outcome of $\mathrm{T}$-cell encounter with antigen would be activation or anergy.

The critical role of costimulation in regulating the immune response has given impetus to the study of costimulation and resulted in incredible growth of this field, since an understanding of costimulation is of fundamental and therapeutic interest. Early studies defined the function of the $\mathrm{CD} 28$ receptor as a stimulatory receptor for the activation of naive T cells (2), and identified the B7 family members, B7-1 (CD80) and B7-2 (CD86) as its ligands (5-7). The interaction between CD28 and B7-1/B7-2 fulfilled many of the requirements for the costimulatory signal postulated by Lafferty, Schwarz, and colleagues (1). The CD28 homolog cytotoxic T lymphocyte-associated antigen-4 (CTLA-4) (3) was found to be a higher affinity binding partner for B7-1 and B7-2, as compared to CD28 (4). It was presumed that CTLA-4 would also be a stimulatory receptor; however, the dramatic fatal inflammatory phenotype of CTLA-4 deficient (-/-) mice revealed the critical inhibitory function of CTLA-4 $(10,11)$. Furthermore, the phenotype of CTLA- $4^{-/}$mice demonstrated that T-cell costimulatory receptors could deliver inhibitory, as well as stimulatory, second signals for T-cell responses, and provided the first indication that inhibitory second signals (co-inhibitory) could regulate T-cell tolerance.

While the B7-1/B7-2:CD28/CTLA-4 pathway is the best characterized T-cell costimulatory pathway, there are now many additional costimulatory pathways. These pathways fall into two major families: the immunoglobulin (Ig) superfamily, which includes the B7/CD28, CD2, and T-cell Ig and mucin domain (TIM) families, and the tumor necrosis family (TNF)/ TNF receptor (TNFR) family. Reviews in this issue of Immunol Rev discuss the functions of pathways within all of these families. These articles not only review the current understanding of costimulation on the fundamental level, but also the therapeutic opportunities and challenges of targeting costimulatory pathways for treating autoimmunity, graft rejection, cancer and infectious diseases. In this introduction, I provide perspectives on

Correspondence to: Arlene H. Sharpe, Department of Pathology, Harvard Medical School, NRB, room 837, 77 Avenue Louis Pasteur, Boston, MA 02115 USA, Tel.: + 1617432 6568, Fax: +1 617432 6570, arlene_sharpe@hms.harvard.edu. 
key functions of costimulatory pathways, highlighting recent advances, and discuss how this progress is changing our view of the functions of T-cell costimulation.

\section{Pathways in the B7/CD28 family}

Despite intensive study, CD28-mediated cosignaling mechanisms are incompletely understood. Novel imaging technologies are providing new insights. Rudd and colleagues (5) review the current knowledge of signaling molecules involved in CD28 function, and the roles of phosphatidylinositol 3-kinase (PI3K), Wiskott-Aldrich syndrome protein (WASP), and SNX9 in the control of CD28 cell-surface expression and endocytosis. Saito and colleagues (6) discuss results of recent dynamic imaging studies which have defined the minimal component of the immunological synapse (IS) as the TCR microcluster, and describe the relationship between TCR and CD28 in microclusters during the process of Tcell activation. These imaging studies reveal the function of CD28 during initial T-cell activation: CD28 localizes coordinately with TCRs to form microclusters; then recruits protein kinase $\mathrm{C} \theta(\mathrm{PKC} \theta)$ to the TCR-CD28 microclusters, resulting in the initial signals for T-cell activation. Interestingly, $\mathrm{CD} 28$ retains $\mathrm{PKC} \theta$ at a spatially unique subregion of the IS, leading to sustained signals for T-cell activation.

While early studies of the B7-1/B7-2:CD28/CTLA-4 pathway focused on its role in controlling the fate decision of naive $\mathrm{T}$ cells between activation and anergy, more recent work has revealed that this pathway not only regulates the responses of naive $\mathrm{T}$ cells, but also is key for the development and function of regulatory T cells (Tregs). Bour-Jordan and Bluestone (7) discuss the functions of CD28 and CTLA-4 as critical regulators of Treg homeostasis and function. CD28 can have dual functions in T-cell biology: CD28 enables the immune system to efficiently mount T-cell responses against microbes but at the same time puts in place regulatory mechanisms needed to terminate immune responses and prevent autoimmunity.

Likewise, CTLA-4 exerts its critical inhibitory functions in multiple ways. Articles by Allison (8), Bluestone (7), and Rudd (5) review cell-intrinsic (affecting only the cells expressing CTLA-4) and cell-extrinsic (affecting other cells) mechanisms of CTLA-4 function. Cell-intrinsic mechanisms include ligand competition and the association of CTLA-4 cytoplasmic tail with signaling components which inhibit TCR signaling. Rudd (5) discusses new data indicating that CTLA-4 can induce positive signaling events that affect cell adhesion, motility, and pro-survival pathways. These signaling studies may help explain microscopy studies that show that CTLA- 4 can control T-cell adhesion and motility. These latter findings have led to the 'reverse-stop signaling model' which provides a mechanism by which CTLA- 4 could raise the threshold for TCR signaling by limiting contact between $\mathrm{T}$ cells and antigen presenting cells (APCs), reversing the TCR-induced signals needed for firm contact between T cells and APCs, and blocking formation of signaling microclusters. There are data to suggest that CTLA-4 may be able to alter APC function by reverse signaling into APCs, leading to production of the immunosuppressive enzyme, indoleamine 2,3 dioxygenase (IDO) or by down-modulating costimulatory molecules on APCs. Although the role of CTLA-4 in Treg biology has been controversial, recent data unambiguously have established that CTLA-4 plays a critical role in the function of Tregs (16). Further mechanistic studies are needed to determine how CTLA-4 controls Tregs.

The B7 and CD28 gene families have grown substantially with mining of the human genome sequence, and the pathways defined by these new members regulate the activation, inhibition and fine-tuning of T-cell responses. A great surprise has been the abundance of inhibitory signals delivered by these new B7/CD28 family members. The newer CD28 family members, inducible T-cell co-stimulator (ICOS), programmed cell death-1 (PD-1), 
and B and T lymphocyte associated (BTLA) are inducibly expressed on T cells and have important roles in controlling the balance between effector and Tregs. PD-1 and BTLA are also expressed on B cells and APCs, and appear to have broader immunoregulatory functions, which necessitate cell type-specific analyses of their functions. The new B7 family members, ICOS ligand (B7h, GL50, B7RP-1, LICOS and B7-H2), PD-L1 (B7-H1), PD-L2 (B7-DC), B7-H3 and B7-H4 (B7x/B7-S1), B7S3, and BTNL2, can be expressed not only on professional APCs, but also on nonhematopoietic cells. The non-hematopoietic cell expression of these B7 family members suggests that they may function to regulate $\mathrm{T}$-cell activation and/or tolerance in non-lymphoid organs.

The ICOS/ICOS ligand pathway has critical roles in stimulating effector T-cell responses and T-dependent B-cell responses, and regulating T-cell tolerance, as reviewed by Dong in this issue (9). While CD28 and ICOS have overlapping functions in early T-cell activation, ICOS has emerged as an important receptor in the immune system to fine tune T-cell effector functions. In addition, ICOS is important for generation of chemochine (C-X-C motif) receptor 5 (CXCR5) ${ }^{+}$follicular helper $\mathrm{T}$ cells (TFH), a unique T-cell subset that regulates germinal center reactions and humoral immunity. Recent studies in $\mathrm{ICOS}^{-1-}$ mice indicate that ICOS can regulate interleukin-21 (IL-21) production, which in turn regulates the expansion of T helper (Th) type 17 (Th17) cells and TFH. ICOS also plays an important role in controlling IL-10 producing Tregs and peripheral T-cell tolerance. Grimbacher and colleagues (10) review the discovery of mutations in ICOS in patients with common variable immunodeficiency. Human ICOS deficiency leads to defective IL-10 and IL-17 production, impaired germinal center-affinity maturation and isotype class switching, which result in profound hypogammaglobulinemia.

Interactions between the inhibitory receptor PD-1 and its ligands, PD-L1 and PD-L2, regulate both the induction and maintenance of peripheral T-cell tolerance [Reviewed by Dong (9) in this issue]. In addition, PD-1 and PD-L1 exert critical inhibitory functions in the setting of persistent antigen expression, as in chronic viral infections and tumors [Reviewed by Allison (8) and Riley (11) in this issue]. Studies in the chronic lymphocytic choriomeningitis virus (LCMV)-infection model provided the first indication that that this pathway contributes to T-cell dysfunction during chronic viral infection (20). PD-1 is overexpressed on exhausted T cells, and immune function in these exhausted T cells is restored upon PD-1 or PD-L1 blockade. This observation has been extended to human immunodeficiency virus (HIV), Simian immunodeficiency virus (SIV), Hepatitis B virus (HBV), and Hepatitis $\mathrm{C}(\mathrm{HCV})$ infections, and has led to new strategies for treating chronic viral infections (12). High PD-L1 expression on tumor cells correlates with poor prognosis in a variety of human cancers, and PD-1 or PD-L1 blockade has been shown to mediate some tumor protection in mice (13). A humanized anti-PD-1 antibody is in clinical trials in cancer patients. Relatively little is known about PD-1 signaling; Riley reviews (11) current understanding of signaling pathways altered by PD-1, and discusses distinct mechanisms by which PD- 1 and CTLA-4 inhibit T cells. One key difference is that PD-1 engagement blocks induction of PI3K activity, whereas CTLA-4 does not. The targeting of distinct signaling molecules by PD- 1 and CTLA- 4 suggests that approaches that block PD- 1 and CTLA-4 signaling should be synergistic.

Understanding of the function of the B7 family member B7-H3 is at an earlier stage. Dong (9) and Chen (14) review data that support stimulatory and inhibitory functions for B7-H3, discuss recent work that has identified TLT-2 (triggering receptor expressed on myeloid cells-like transcript 2) as a receptor for $\mathrm{B} 7-\mathrm{H} 3$, and entertain the idea that an additional receptor-mediating inhibitory responses for B7-H3 may exist. There is consensus that B7H4 functions as a co-inhibitory molecule, and Dong (9), Chen (14), and Allison (8) and their colleagues review the inhibitory functions of B7-H4 (B7x. B7S1). Yi and Chen (14) discuss 
new data that B7-H4 serves as an important negative regulator of innate immunity through inhibition of neutrophil growth. Functional studies of B7S3 and BTNL2 are limited, but indicate that both can inhibit T-cell activation. Mutations in the human BTNL2 gene have been identified, which are linked with sarcoidosis, and polymorphisms strongly associated with several human autoimmune diseases (24), suggesting that BTNL2 may serve to regulate tolerance or inflammation.

\section{Pathways in the TNFR/TNF family}

TNFR family members can recruit TNF receptor associated factor (TRAF) adapter proteins and activate the nuclear factor- $\kappa \mathrm{B}(\mathrm{NF}-\mathrm{\kappa B})$ signaling pathway, making them fundamentally distinct from costimulators such as CD28 or ICOS. CD40 and its ligand, CD154, were the first costimulatory molecules to be identified as members of the TNFR/TNF superfamily, and are crucial for the functions of B cells and dendritic cells (DCs). CD40 signals into DCs, and B cells as well as other cell types, including tumor cells. Noelle and colleagues (15) discuss downstream signaling pathways initiated by CD40 through TRAF proteins, and the essential functions of CD4/CD40L interactions in controlling humoral, cellular, and tumor immunity (15).

Studies of the CD27/CD70, CD30/CD30L, OX40/OX40L, 4-1BB/4-1BBL, glucocorticoidinduced TNF receptor (GITR)/GITR ligand (GITRL), herpes virus entry mediator (HVEM)/ lymphotoxin-like, exhibits inducible expression, and competes with herpes simplex virus glycoprotein D for HVEM, a receptor expressed by T lymphocytes (LIGHT) pathways indicate that these TNFR/TNF family members can provide important costimulatory signals. With the exception of CD27, which is constitutively expressed on naive T cells, the other TNFR are expressed only upon T-cell activation. In addition, memory $\mathrm{T}$ cells and Tregs constitutively express certain family members. These expression patterns have suggested that the TNFR/TNF family may be important in controlling effector and memory responses.

Recent work indicates that OX40/OX40L and 4-1BB/4-1BBL interactions have key roles in regulating the balance between effector and Treg responses. As reviewed by Croft (16) in this issue, OX40 promotes effector T-cell expansion and survival, as well as the generation, reactivation, and maintenance of memory T cells. OX40 also blocks natural Treg activity, and antagonizes the generation of inducible Tregs. Thus, OX40 can promotes effector and memory T-cell responses directly by stimulatory these cells and indirectly by inhibiting Tregs. OX40 has become an attractive therapeutic target; OX40 blockade can attenuate autoimmunity and inflammation, while stimulating OX40 can enhance anti-tumor immunity. As reviewed by Watts (17) in this issue, the major role of 4-1BB is for survival of activated and memory $\mathrm{T}$ cells, with preferential effects on $\mathrm{CD} 8^{+} \mathrm{T}$ cells. However, when other costimulatory signals are limiting, 4-1BB signals can cooperate with TCR-induced signals to enhance proliferation and development of effector function, perhaps by allowing $T$ cells to survive through rounds of division. In addition, 4-1BB can exert immunosuppressive effects in some settings. Agonistic anti-4-1BB mAbs not only can expand CD8 ${ }^{+} \mathrm{T}$ cells, but also inhibit humoral immunity and ameliorate autoimmunity. 4-1BB is expressed on Tregs, and ligation of 4-1BB on Tregs can enhance their expansion and possibly their suppressive function. 4-1BB also can induce interferon- $\gamma(\mathrm{IFN}-\gamma)$ and $\mathrm{CD}^{+} \mathrm{T}$-cell-dependent suppression. Further work is needed to understand how 4-1BB controls the balance between effector and regulatory cell function. This is a clinically relevant issue, as agonistic anti-4-1BB mAbs are in clinical trials.

The CD27/CD70 pathway also is important for generation of effector and memory T-cell responses and promotes proliferation and survival of activated $\mathrm{T}$ cells [Reviewed by van Lier (18) in this issue]. The potency of costimulation by CD27:CD70 interactions is 
illustrated by the development of immunopathology in transgenic mice that constitutively express CD70 or CD27. These findings give impetus to studies examining the impact of CD70:CD27 interactions during chronic antigen stimulation, such as chronic virus infection and autoimmunity.

HVEM sits at a pivot point, connecting the TNFR and Ig superfamilies with a complicated molecular cross-talk among its receptors: LIGHT, lymphotoxin $\alpha$ (LT $\alpha)$, CD160, and BTLA. LIGHT can influence T-cell activation both directly and indirectly by engaging receptors expressed on T cells and other cell types. The binding of LIGHT or LT $\alpha$ to HVEM delivers a costimulatory signal [Reviewed by $\mathrm{Fu}(19)$ in this issue] whereas the binding of BTLA or CD160 to HVEM delivers a coinhibitory signal [Reviewed by Freeman (20) in this issue]. Thus HVEM is a bidirectional switch regulating T-cell activation in a costimulatory or coinhibitory fashion depending upon which ligand is engaged. LIGHT or $L T \alpha_{1} \beta_{2}$ binding to lymphotoxin- $\beta$ R (LT $\beta R$ ), which is expressed by DCs and stromal cells in lymph nodes and other tissues, induces chemokine production by stromal cells resulting in development of lymphoid structures and DC expansion. Thus, LIGHT has the capacity to serve as both a costimulatory ligand for $\mathrm{T}$ cells and a stromal activator to induce chemokine production and lymphoid recruitment. For these reasons, it has become an attractive target for immunotherapy and methods for intratumoral delivery of LIGHT are being studied. Freeman (20) discusses the inhibitory consequences of HVEM interactions with CD160 and BTLA and provides insight into how engagement of HVEM can lead to stimulatory or inhibitory outcomes. HVEM has distinct domains for binding its stimulatory ligand LIGHT and its coinhibitory ligands CD160 and BTLA. Therapies targeting the cysteine-rich domain-1 of HVEM to block BTLA and CD160 binding are being developed to enhance immune responses and vaccination.

\section{Pathways in the TIM family}

The functions of the TIM gene family are just emerging. The TIM family initially was identified in searches for Th1-specific or asthma-associated genes and thought to regulate Th1/Th2 polarization. Recent work indicates that TIM family members have far broader immunoregulatory functions that impact innate and adaptive immunity. New functions of the TIM family have been revealed by the recent identification of TIM- 1 and TIM- 4 as phosphatidylserine receptors and their roles in clearance of apoptotic cells $(31,32)$. Kuchroo, DeKruyff, and Umetsu (21) discuss the diverse functions of TIM family members in regulating T-cell activation, induction of T-cell apoptosis, T-cell tolerance, and clearance of apoptotic cells. While the understanding of the TIM family is at an early stage, the clinical significance of this family is illustrated by the identification of TIM-1 as a potent asthma and allergy susceptibility gene, and by the impact of targeting TIM family members in models of asthma, autoimmunity and transplantation.

\section{Translation of costimulation to the clinic}

The multitude of T-cell costimulatory pathways has important therapeutic implications. As discussed by Sayegh (22) and colleagues, knowledge of which, where, and when specific costimulatory pathways are most critical for controlling anti-donor T-cell responses is necessary for designing effective therapies to modulate these pathways to prevent graft versus host disease, graft rejection, and induce transplant tolerance. It will be important to understand the unique and overlapping roles of costimulatory pathways for controlling effector $\mathrm{T}$ cells, memory $\mathrm{T}$ cells, and Treg responses after transplantation. Since activated effector/memory $\mathrm{T}$ cells and Tregs often can express similar costimulatory molecules, development of selective approaches to block effector/memory T cells, but not Tregs, poses some challenges. In addition, detailed analyses of receptors and ligands in each pathway are 
needed to guide the choice of the receptor or ligand as a therapeutic target, as discussed by Larsen (23). Finally, an understanding of the similarities and differences of cosimulatory pathways in rodents, non-human primates and humans is critically important for the successful translation of costimulatory pathways into new therapeutics, illustrated in this issue by discussions of the bench-to-benchside translation of costimulatory blockade for the CD28/B7, CD154/CD40, and lymphocyte function-associated antigen 1 (LFA-1)/ intracellular adhesion molecules (ICAM) pathways $(23,24)$.

Many of the issues of costimulatory modulation are similar for treatment of transplant rejection and autoimmunity, since the goals are to prevent pathogenic T-cell responses and induce tolerance. While animal models have revealed multiple roles of costimulation and coinhibition in autoimmunity, genetic studies in humans also are now identifying costimulatory molecules associated with risk to human autoimmunity and genetic variants that confer risk to human autoimmune diseases. As reviewed by Maier and Hafler (25), genome wide association scans have identified convincing associations of CTLA-4, CD58, CD40, ICOSL, CD244, CD226, PDCD1 (PD-1), and TNF superfamily member 4 (TNFSF4, OX40L) with human autoimmune diseases. Understanding the functions of these genes and their variants has the potential to provide insights in disease pathogenesis and therapy. Therapeutic approaches for autoimmunity that focus on inhibition of signal one (delivery of suboptimal TCR stimulation in antigen specific therapy by ethylene carbodiimide (ECDI)coupled APC treatment) or signal two (modulation of the B7:CD28/CTLA-4 pathway) are in clinical trials [Reviewed by Miller (26) and Nadler (24) in this issue]. A critical challenge is to find the optimal balance between targeting activation and effector functions of selfreactive $\mathrm{T}$ cells without compromising anti-microbial immunity or increasing susceptibility of the patient to development of cancer. However, the therapeutic potential of costimulatory pathway modulation is beginning to be realized with translation of preclinical studies on CD28 into clinical practice. CTLA-4Ig (Abatacept) is now an US Food and Drug Administration (FDA)-approved therapy for rheumatoid arthritis.

For tumor immunotherapy, there are multiple barriers to the anti-tumor response, and coinhibitory pathways participate in shielding tumors from immune eradication. The differential expression of coinhibitory molecules (such as B7-H4) on certain cancers provides an opportunity for selective immunotherapeutic intervention. Interference with multiple coinhibitory pathways may be needed for optimal therapeutic benefit. Engaging costimulatory pathways, as well as blocking coinhibitory pathways, has therapeutic potential for treatment of cancer, as discussed by Gajewski (13) and Allison (8). However, challenges are posed by the development of inflammation or autoimmunity following immune intervention. Gajewski (13) reviews pathways with the highest potential for clinical application and translation to the clinic, summarizing clinical trials aimed at boosting positive costimulation and anti-tumor immunity with agonistic CD40, 4-1BB, or OX40 antibodies, and clinical trials that block the coinhibitory receptors CTLA-4 and PD-1.

Coinhibitory pathways also have become therapeutic targets for combating chronic viral infections. Blockade of the PD-1/PD-L pathway identified the key role of inhibitory receptors in mediating T-cell exhaustion. Exhausted $\mathrm{CD}^{+} \mathrm{T}$ cells express multiple inhibitory receptors, including PD-1, lymphocyte-activation gene 3 (LAG-3), CD160, 2B4 (CD244), and CTLA-4 (39). The coexpression of multiple inhibitory receptors has been associated with greater T-cell exhaustion and more severe infection. Co-blockade of PD-1 and LAG-3 synergistically improved T-cell responses and reduced viral load, demonstrating that distinct inhibitory pathways can uniquely regulate T-cell exhaustion (39). Further studies are needed to define the unique and overlapping functions of coinhibitory pathways in exhausted T cells and to test the therapeutic benefits of targeting multiple coinhibitory pathways during chronic viral infection. 
Recent technological advances in structural biology provide new opportunities for understanding the structural and chemical features of costimulatory receptors and ligands, and their mechanisms of costimulation. Almo and colleagues (27) discuss the crystal structures of costimulatory receptors and ligands in the Ig, TNF, and TIM families, and illustrate how structural understanding can provide important mechanistic insights through defining chemical and physical features that underlie receptor/ligand specificity, affinity, and oligomeric state. These approaches also may guide the development of improved therapeutic agents and reveal new therapeutic strategies for targeting costimulatory pathways.

In summary, our understanding of costimulation has evolved significantly from the two signal model for T-cell activation. There are both stimulatory and inhibitory second signals, and the inhibitory signals play important roles in the induction and maintenance of T-cell tolerance. In addition, we now realize that costimulatory pathways not only regulate the initial activation of naive $\mathrm{T}$ cells but also control effector, memory and regulatory $\mathrm{T}$ cells. These pathways may control tolerance by preventing effector T-cell responses and by promoting/maintaining Tregs. Costimulatory and coinhibitory molecules can be expressed on non-hematopoietic cells as well as professional APCs, and expression on parenchymal cells may regulate T-cell responses in specific microenvironments. Although costimulatory molecules are generally viewed through their effects on T cells, many receptor ligand interactions may lead to bidirectional signaling with important consequences for the non Tcell partner. Thus, molecular definition of T-cell costimulatory pathways is providing insights into the exquisite regulation of T-cell activation and tolerance. Cosignaling pathways are major regulators of the critical balance between tolerance and immune responses to pathogens that are effective with an acceptable level of tissue damage.

Although significant progress has been made, many questions still remain. First, there are still many orphan molecules whose unidentified ligands are the key to understanding their functions. The identification of phosphatidyserine as a ligand for TIM-1 and TIM-4 remind us that not all these molecules will have protein ligands. Second, with the growth in the number of costimulatory pathways, further work is needed to understand which pathways provide overlapping positive and negative signals and whether there is a hierarchy in the orchestration of these signals. In particular, we do not understand why there are so many coinhibitory molecules. The large number of coinhibitory molecules suggests that these molecules may have selective functions in regulating tolerance. Third, we understand little about the function of costimulatory molecules in non-lymphoid organs. Recent work indicates that PD-L1 on non-hematopoietic cells can regulate tissue tolerance (28), giving impetus to investigations of other B7 family members on non-hematopoietic cells in regulating immune responses in tissue microenvironments. Finally, many new costimulatory molecules have receptors on non-T cells, suggesting that these molecules may have broader immunoregulatory roles. The therapeutic potential of additional understanding of T-cell costimulatory pathways is underscored by the FDA approval of CTLA-4-Ig for rheumatoid arthritis and the clinical trials in progress with other modulators of costimulation.

\section{Acknowledgments}

This work was supported by grants from the National Institutes of health

\section{References}

1. Lafferty KJ, Andrus L, Prowse SJ. Role of lymphokine and antigen in the control of specific T cell responses. Immunol Rev. 1980; 51:279-314. [PubMed: 7000674] 
2. June CH, Ledbetter JA, Linsley PS, Thompson CB. Role of the CD28 receptor in T-cell activation. Immunol Today. 1990; 11:211-216. [PubMed: 2162180]

3. Brunet JF, et al. A new member of the immunoglobulin superfamily--CTLA-4. Nature. 1987; 328:267-270. [PubMed: 3496540]

4. Linsley PS, Brady W, Urnes M, Grosmaire LS, Damle NK, Ledbetter JA. CTLA-4 is a second receptor for the B cell activation antigen B7. J Exp Med. 1991; 174:561-569. [PubMed: 1714933]

5. Rudd CE, Taylor A, Schneider H. CD28 and CTLA-4 coreceptor expression and signal transduction. Immunol Rev. 2009; 229

6. Yokosuka T, Saito T. Dynamic regulation of T-cell costimulation through TCR-CD28 microclusters. Immunol Rev. 2009; 229

7. Bour-Jordan H, Bluestone JA. Regulating the regulators: costimulatory signals control the homeostasis and function of regulatory T cells. Immunol Rev. 2009; 229

8. Pentcheva-Hoang T, Corse E, Allison JP. Negative regulators of T-cell activation: potential targets for therapeutic intervention in cancer, autoimmune disease, and persistent infections. Immunol Rev. 2009; 229

9. Nurieva RI, Liu X, Dong C. Yin-Yang of costimulation: crucial controls of immune tolerance and function. Immunol Rev. 2009; 229

10. Yong PFK, Salzer U, Grimbacher B. The role of costimulation in antibody deficiencies: ICOS and common variable immunodeficiency. Immunol Rev. 2009; 229

11. Riley JL. PD-1 signaling in primary T cells. Immunol Rev. 2009; 229

12. Sharpe AH, Wherry EJ, Ahmed R, Freeman GJ. The function of programmed cell death 1 and its ligands in regulating autoimmunity and infection. Nat Immunol. 2007; 8:239-245. [PubMed: 17304234]

13. Driessens G, Kline J, Gajewski TF. Costimulatory and coinhibitory receptors in anti-tumor immunity. Immunol Rev. 2009; 229

14. Yi KH, Chen L. Fine tuning the immune response through B7-H3 and B7-H4. Immunol Rev. 2009; 229

15. Elgueta R, Benson MJ, de Vries VC, Wasiuk A, Guo Y, Noelle RJ. Molecular mechanism and function of CD40/CD40L engagement in the immune system. Immunol Rev. 2009; 229

16. Croft M, So T, Duan W, Soroosh P. The significance of OX40 and OX40L to T-cell biology and immune disease. Immunol Rev. 2009; 229

17. Wang C, Lin GHY, McPherson AJ, Watts TH. Immune regulation by 4-1BB and 4-1BBL: complexities and challenges. Immunol Rev. 2009; 229

18. Nolte MA, van Olffen RW, Van Gisbergen KPJM, van Lier RAW. Timing and tuning of CD27CD70 interactions: the impact of signal strength in setting the balance between adaptive responses and immunopathology. Immunol Rev. 2009; 229

19. Wang Y, Zhu M, Miller M, Fu YX. Immunoregulation by Tumor Necrosis Factor Superfamily Member LIGHT. Immunol Rev. 2009; 229

20. Cai G, Freeman GJ. The CD160, BTLA, LIGHT/HVEM pathway: a bidirectional switch regulating T-cell activation. Immunol Rev. 2009; 229

21. Rodriguez-Manzanet R, DeKruyff R, Kuchroo VK, Umetsu DT. The costimulatory role of TIM molecules. Immunol Rev. 2009; 229

22. Li XC, Rothstein DM, Sayegh MH. Costimulatory pathways in transplantation: challenges and new developments. Immunol Rev. 2009; 229

23. Ford ML, Larsen CP. Translating costimulation blockade to the clinic: lessons learned from three pathways. Immunol Rev. 2009; 229

24. Linsely PS, Nadler SG. The clinical utility of inhibiting CD28-mediated costimulation. Immunol Rev. 2009; 229

25. Maier LM, Hafler DA. Autoimmunity risk alleles in costimulation pathways. Immunol Rev. 2009; 229

26. Podojil JR, Miller SD. Molecular mechanisms of T-cell receptor and costimulatory molecule ligation/blockade in autoimmune disease therapy. Immunol Rev. 2009; 229 
27. Chattopadhyay K, Lazar-Molnar E, Yan Q, Rubinstein R, Zhan C, Vigdorovich V, Ramagopal UA, Bonanno J, Nathenson SG, Almo SC. Sequence, structure, function, immunity: structural genomics of costimulation. Immunol Rev. 2009; 229

28. Keir ME, Butte MJ, Freeman GJ, Sharpe AH. PD-1 and its ligands in tolerance and immunity. Annu Rev Immunol. 2008; 26:677-704. [PubMed: 18173375] 